\title{
Corrigendum to "Time and Covariance Threshold Triggered Optimal Uncooperative Rendezvous Using Angles-Only Navigation"
}

\author{
Yue You ${ }^{D},{ }^{1}$ Hua Wang $\left(\mathbb{D},{ }^{1}\right.$ Christophe Paccolat $\mathbb{D}^{\mathbb{D}},{ }^{2}$ Volker Gass $\mathbb{D}^{\mathrm{D}},{ }^{2}$ Jean-Philippe Thiran, ${ }^{3}$ \\ and Jiu Ren $\operatorname{Li}{ }^{4}{ }^{4}$

\footnotetext{
${ }^{1}$ The College of Aerospace Science and Engineering, National University of Defense Technology, Changsha 410073, China

${ }^{2}$ Swiss Space Center, École Polytechnique Fédérale de Lausanne, 1015 Lausanne, Switzerland

${ }^{3}$ Signal Processing Lab (LTS5), École Polytechnique Fédérale de Lausanne, 1015 Lausanne, Switzerland
} \\ ${ }^{4}$ The Manned Space Technology System Center, Beijing 100094, China
}

Correspondence should be addressed to Yue You; youyue_nudt@126.com

Received 13 June 2018; Accepted 27 June 2018; Published 30 September 2018

Copyright ( 2018 Yue You et al. This is an open access article distributed under the Creative Commons Attribution License, which permits unrestricted use, distribution, and reproduction in any medium, provided the original work is properly cited.

The article titled "Time and Covariance Threshold Triggered Optimal Uncooperative Rendezvous Using Angles-Only Navigation" [1], is an extended version of the authors' conference article, "You Yue, Wang Hua, C. Paccolat and Li Jiu Ren, "Time and Covariance Threshold Triggered Optimal Uncooperative Rendezvous Using Angles Only Navigation," 2016 IEEE Chinese Guidance, Navigation and Control Conference (CGNCC), Nanjing, 2016, pp. 943-948. doi:10.1109/ CGNCC.2016.7828912," which was published after the article was submitted and was not cited [2].

The abstracts are different, the introduction was extended, 10 references were added, more detail is given in "Covariance Threshold Triggered Maneuver Planning," a discussion on "earth eclipse rate influence" was added, and two figures were added, Figures 5 and 6.

This was raised by the first author, who apologizes for not declaring this previous version of the work. The editorial board agreed to publish a corrigendum.

\section{References}

[1] Y. You, H. Wang, C. Paccolat, V. Gass, J.-P. Thiran, and J. R. Li, "Time and covariance threshold triggered optimal uncooperative rendezvous using angles-only navigation," International Journal of Aerospace Engineering, vol. 2017, Article ID 5451908, 10 pages, 2017.
[2] Y. Yue, W. Hua, C. Paccolat, and J. R. Li, "Time and covariance threshold triggered optimal uncooperative rendezvous using angles only navigation," in 2016 IEEE Chinese Guidance, Navigation and Control Conference (CGNCC), pp. 943-948, Nanjing, China, August 2016. 


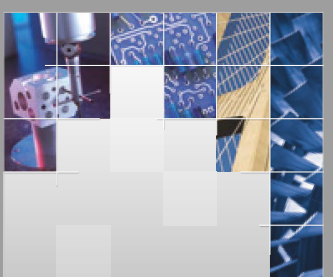

\section{Enfincering}
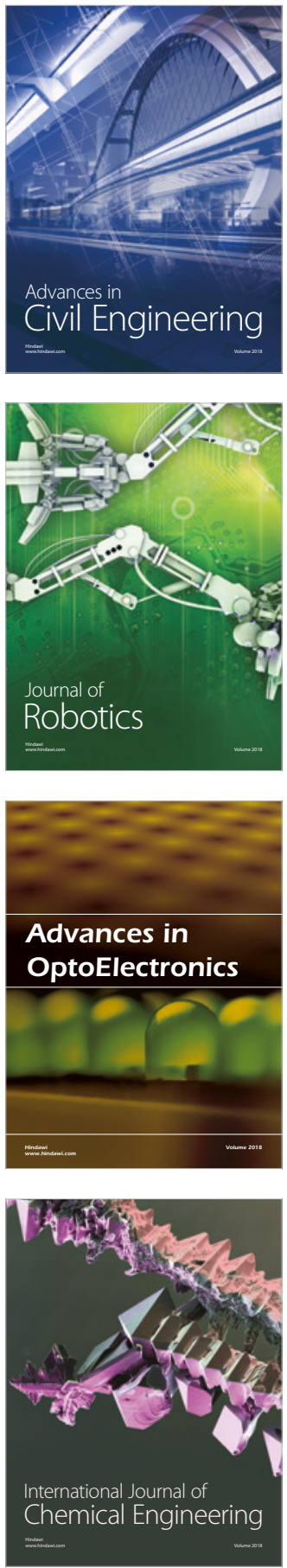

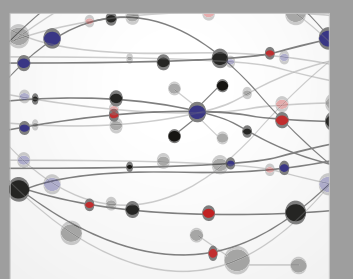

\section{Rotating \\ Machinery}

The Scientific World Journal

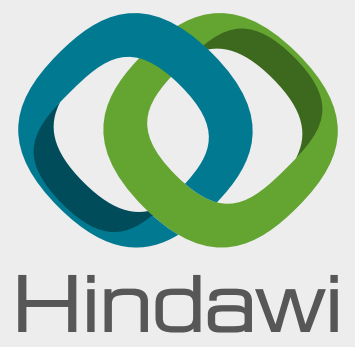

Submit your manuscripts at

www.hindawi.com
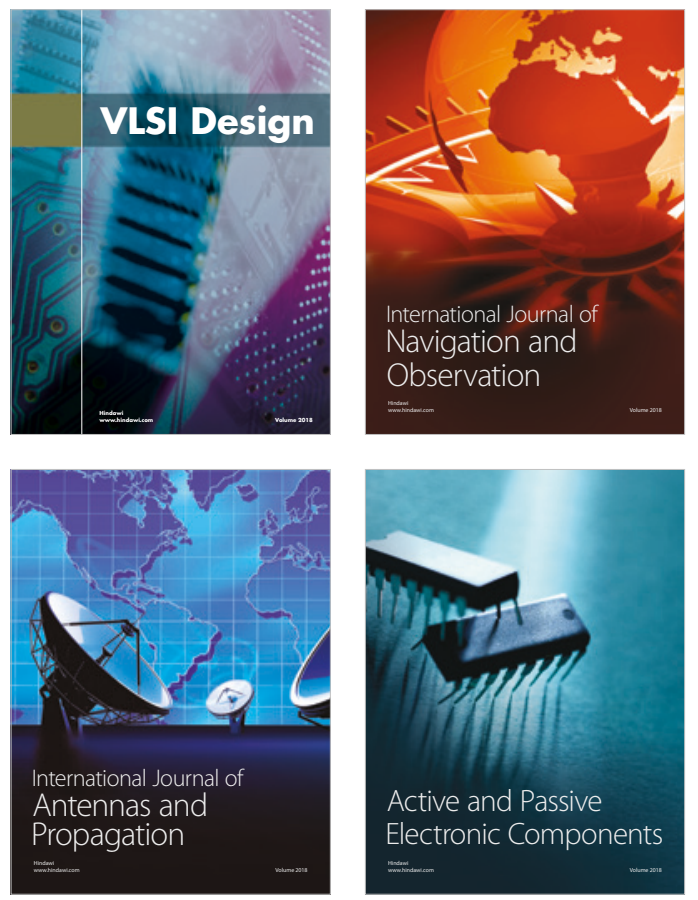
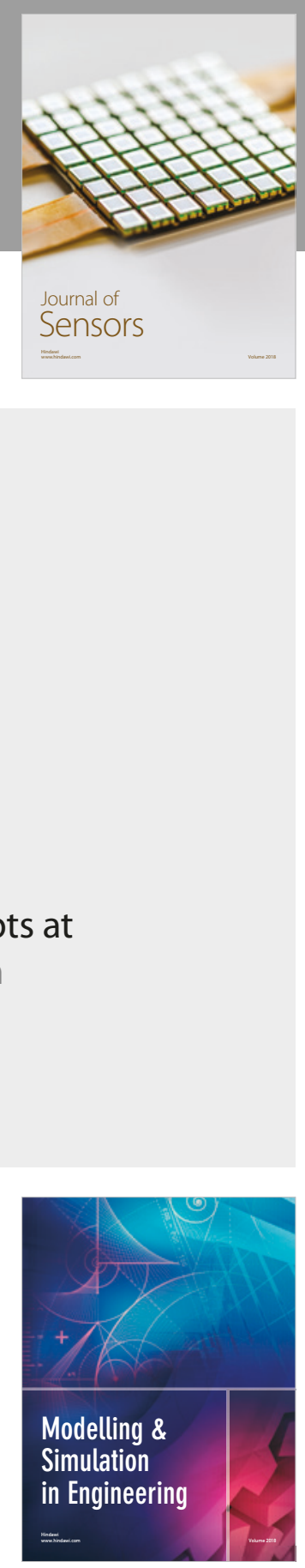

\section{Advances \\ Multimedia}
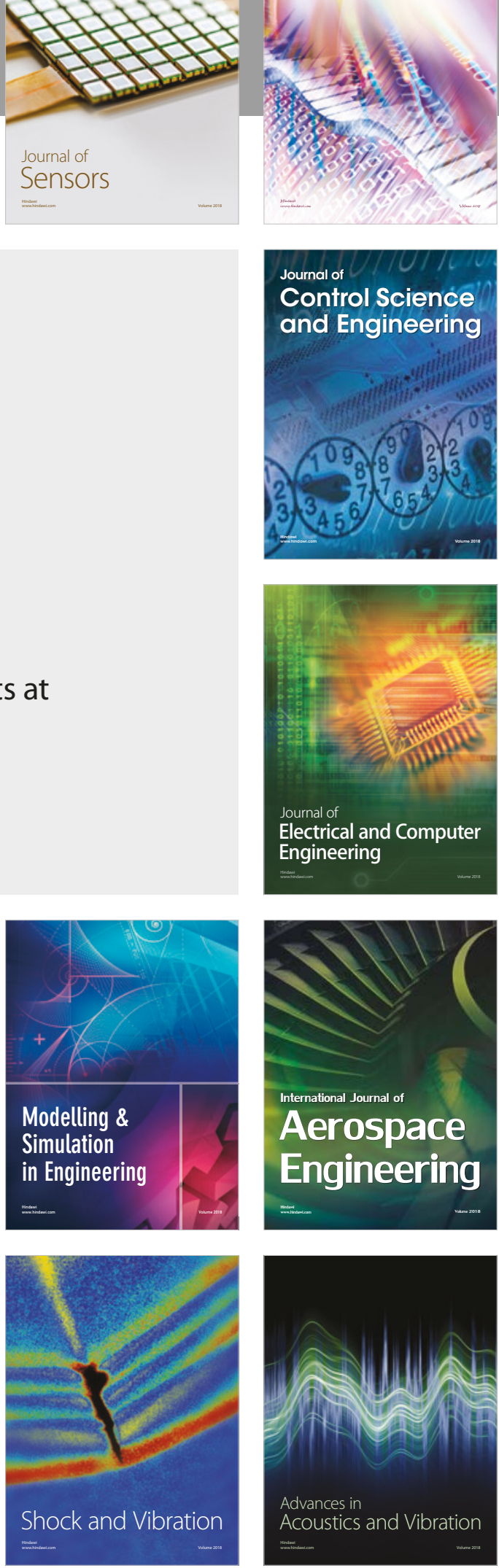